\section{Towards a Theory of Cell Assemblies}

\author{
G. Palm
}

Max-Planck-Institut für biologische Kybernetik, Tübingen, FRG between cell assemblies. In this way we may also form new cell assemblies.

4. How can we describe the electrical activity of all the neurons in the brain in a simplified way? Answer: As a succession of active cell assemblies governed by the rules of threshold control (cf. Braitenberg, 1978).

It seems that thinking in terms of cell assemblies may provide an intermediate description of the brain between the psychological and the electrophysiological level and with reasonably strong relations to both levels. These strong relations to both levels have lead to the question: Are they real?

From the anatomical point of view the idea of cell assemblies requires excitatory connections between all parts of the cortex (since all kinds of things can be associated with each other) and though the evidence for this is still incomplete there are indications that this is true (cf. Braitenberg, 1978).

From the physiological point of view the idea requires variable excitatory synapses that obey Hebb's rule (i.e. the connectivity is enhanced by coincident pre- and postsynaptic activity). This requirement is hardly accessible to direct experimental investigation, at least in man or higher vertebrates, and there is (to my knowledge) no evidence against it (compare also Cowan, 1976). Furthermore, it would be of interest to the physiologist how large cell assemblies should usually be, i.e. of how many cells they should be formed.

From the psychological point of view we need a very large number of cell assemblies, since we have a large number of concepts.

For example, cell assemblies can be regarded as the neuronal substrate for the "nodes" in a "semantic network" (like the one of Quilian, 1967, 1968). The flow of "activity" through such a network is somehow determined by the "links" in the network [the exact rules have not yet been worked out in the literature, as can be seen from the discussion in Collins and Loftus

3. How do we learn? Answer: By forming association between concepts embodied in excitatory connections 


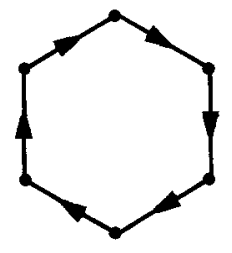

a

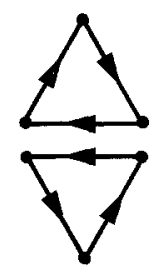

b
Fig. 1a and b. Both networks, a and b, consist of 6 elements and 6 connections. They both are homogeneous: Each element gives and receives one connection. Network $\mathbf{b}$ consists of two assemblies

(1975)]. This flow of activity corresponds to the succession of active cell assemblies in the cortex. Some first speculations on the mechanisms that control the succession of cell assemblies can be found in Braitenberg (1978). As for possible "links" between assemblies, one might consult Sect. 7.

But first of all we have to find out whether there is enough room in the brain for a sufficiently large number of cell assemblies. The problem concerning the number of cell assemblies is of course somehow connected to the question of their size.

This problem has already been tackled in some papers (e.g. Legendy, 1967, 1968). But I think it should be studied more systematically. In this article I want to suggest a mathematical formalism for the statement and investigation of this problem. In the first section I will try to present the problem more clearly; the following sections are devoted to an exact mathematical formulation.

\section{Is the Cortical Network Sufficient to Contain all our Concepts?}

Let me start with a rough picture of the cortical connectivity. The most striking feature of the cortical network is its homogeneity: in particular, every neuron receives and gives roughly the same number of connections. As has already been pointed out in Palm and Braitenberg (1979) it is important to distinguish the cortico-cortical connections in the "grey matter" from those through the "white matter". The first are local connections in one and the same area of the cortex and they are "metrical" in the sense that the chance of a connection between two neurons is dependent on their distance, being highest if the neurons are closest.

The second are global connections between different cortical areas and they are "ametrical" in the sense that the connection between two cells is not dependent on their distance in any simple way. It is now generally believed that inhibition is prominent in the local connections through inhibitory interneurons (although there is of course also local excitation through axon collaterals of pyramidal cells), whereas the global connections (which are the axons of the pyramidal cells) are excitatory. The various "areas" in the brain can perhaps be viewed functionally as "regions of uniform context" (Braitenberg, 1974; Palm and Braitenberg, 1979), which can be interpreted as follows: Inside any one region (corresponding to one context) inhibitory local connections will help to differentiate between concepts (think of the literal meaning of "definition"), whereas the excitatory global connections serve to form the concepts in the first place (think of the literal meaning of "concept").

This interpretation of the cortical connectivity implies that cell assemblies are bound together mainly by the excitatory ametric long range connections and that they consist of cells distributed all over the cortex.

Therefore we can discard metrical considerations and inhibitory connections in the basic formulation of the problem (of the number of cell assemblies).

A cell assembly is a set of neurons that contribute more excitation to each other than they contribute to the average neuron.

Given the homogeneity of the cortical network, the first question is whether an assembly is possible in the cortex at all.

If we have a homogeneously and randomly connected network of $N$ neurons, and each neuron has a probability $p$ of firing, then the total postsynaptic activity $a$ a fixed neuron $A$ receives from the network, is a random variable. Let us now assume that $A$ fires if the total postsynaptic activity $a$ in its dendritic tree exceeds a threshold $\theta$, then there is exactly one value $\theta_{p}$ for $\theta$ which yields again $p$ as the probability of $A$ to fire, i.e. there is exactly one number $\theta_{p}$ such that $\operatorname{pr}\left[a>\theta_{p}\right]=p$. Thus to have a constant average activity of $p \cdot N$ neurons in the network, the average neuron has to have a threshold of $\theta=\theta_{p}$. If $\theta>\theta_{p}$ in the average neuron, the probability of firing will decrease which leads to a decrease in the postsynaptic activity and therefore to a further decrease in the probability of firing, and so on. Finally the activity will die out completely. If $\theta<\theta_{p}$, we get the opposite effect, leading finally to a complete activity of the whole network. In other words, the constant activity at level $N \cdot p$ is unstable and in particular very sensitive to changes of the threshold $\theta$.

Computer simulations of randomly connected neuron networks as well as detailed statistical investigations on neurodynamics (Harth, 1970; Amari, 1974, 1977) have shown that it is easily possible to obtain a constant activity at some nontrivial level $p \cdot N(0<p<1)$ in a randomly connected network and that this level does not change for small variations of $\theta$. This means that the activity in these randomly connected networks is maintained in some subset of the set 


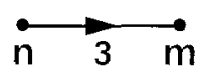

a

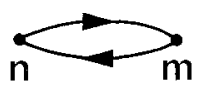

f

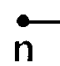<smiles>C1CCCCC1</smiles>

g

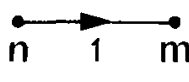

C

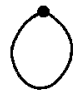

h

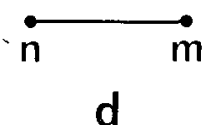

d

Fig. 2

of all the $N$ neurons. The neurons in this subset give more excitation to each other than to the rest (to sustain slight increase of $\theta$ and to avoid total excitation for slight decrease of $\theta$ ).

Moreover, if we define a network as homogeneous, when every element receives and gives the same number of connections, then it is quite trivial to construct homogeneous networks containing such assemblies (Fig. 1).

In summary, it is possible that homogeneous networks contain assemblies, they even often occur in randomly connected networks.

Now only the one suspicion may be left that in a homogenous network the number of assemblies cannot be very large. To analyse this problem in detail we shall employ the mathematical language of graph theory (cf. Harari, 1969). A graph is just a set of points with some arrows or lines connecting them, like Fig. 1 above.

\section{Weighted Graphs}

To describe the dynamics of the cortical network, it is necessary to know the connectivity of the cortex. One could describe this connectivity by the so-called connectivity (or adjacency) matrix, i.e. by numbering the neurons and denoting by $c_{i j}$ the connectivity from neuron $i$ to neuron $j$.

The appearance of this matrix depends to a certain degree on the way the neurons are numbered. The connectivity is given in its purest way as a mapping $c: G \times G \rightarrow \mathbb{R}$, where $G$ is the set of all neurons and $c(n, m)$ gives the strength of connectivity from neuron $n$ to neuron $m$. As usual positive values of $c(n, m)$ mean an excitatory connection, negative values an inhibitory connection, and the value 0 means no connection at all.

A nice way to picture such a connectivity mapping $c$ is by means of a graph: the neurons are denoted as points and the connections are indicated by arrows, e.g. $c(n, m)=3$ is denoted as in Fig. 2a. Arrows with 0 connectivity are, of course, not drawn. For $c(n, m)=1$ we might also write Fig. $2 b$ instead of Fig. 2c. For $c(n, m)=c(m, n)=1$ we may even write Fig. $2 \mathrm{~d}$ or e instead of Fig. 2f. For $n=m, c(n, m)=5$ is denoted as

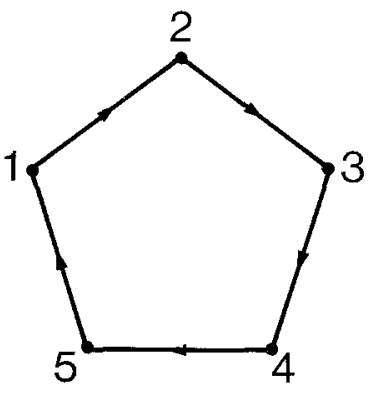

Fig. 3

a

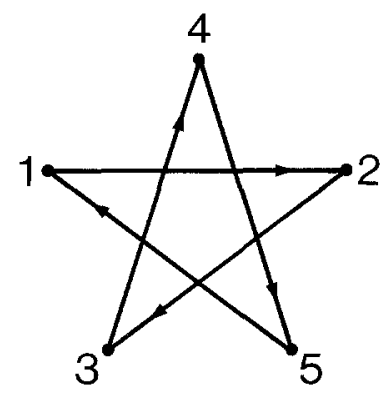

b
Fig. $2 \mathrm{~g}$, and $c(n, m)=1$ simply as Fig. 2h. Such a connection is called a self-connection or loop.

For example the mapping

$c: G \times G \rightarrow\{0,1\}, G=\{1,2,3,4,5\}$

with

$c(1,1)=0 \quad c(2,1)=0 \quad c(3,1)=0 \quad c(4,1)=0 \quad c(5,1)=1$

$c(1,2)=1 \quad c(2,2)=0 \quad c(3,2)=0 \quad c(4,2)=0 \quad c(5,2)=0$

$c(1,3)=0 \quad c(2,3)=1 \quad c(3,3)=0 \quad c(4,3)=0 \quad c(5,3)=0$

$c(1,4)=0 \quad c(2,4)=0 \quad c(3,4)=1 \quad c(4,4)=0 \quad c(5,4)=0$

$c(1,5)=0 \quad c(2,5)=0 \quad c(3,5)=0 \quad c(4,5)=1 \quad c(5,5)=0$

can be described by the connectivity matrix

$C=\left(\begin{array}{lllll}0 & 1 & 0 & 0 & 0 \\ 0 & 0 & 1 & 0 & 0 \\ 0 & 0 & 0 & 1 & 0 \\ 0 & 0 & 0 & 0 & 1 \\ 1 & 0 & 0 & 0 & 0\end{array}\right)$

and can be pictured as in Fig. 3a or b.

Definition. A weighted graph is a pair $\langle G, c\rangle$, where $G$ is a set and $c: G \times G \rightarrow \mathbb{R}$ a mapping. The elements of $G$ are called points. For $x, y \in G, c(x, y)$ is called the strength or weight of the connectivity from $x$ to $y$.

If $c: G \times G \rightarrow\{0,1\}$ and $c(x, x)=0$ for every $x \in G$, $\langle G, c\rangle$ is called a directed graph. If in addition $c(x, y)=c(y, x)$ for every $x$ and $y$ in $G,\langle G, c\rangle$ is called a graph.

Thus graphs, as well as directed graphs, do not contain loops.

I want to use such a graph as a model for the flow of activity in the brain. This can be done in the 


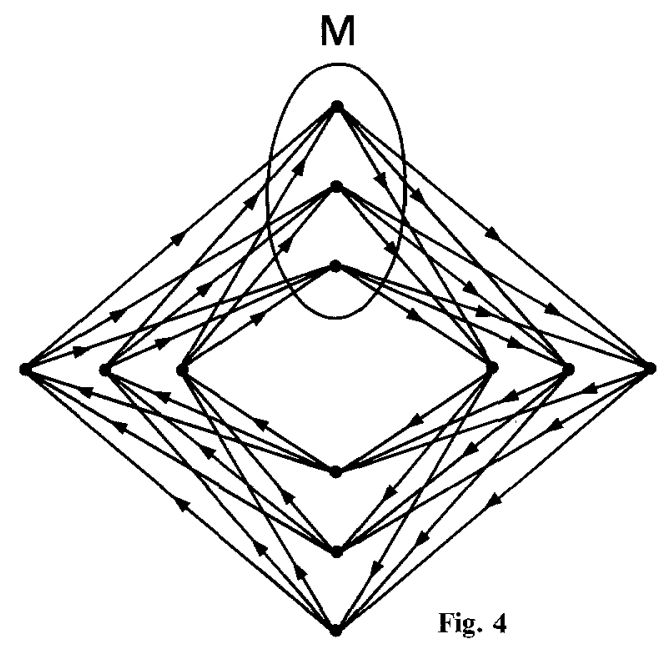

following way. The points are identified with the neurons and $c(x, y)$ gives the total strength of the synapses from neuron $x$ to neuron $y$. Starting from a set $M$ of "on"-neurons, we get the resulting flow of activity through the graph at threshold $\theta$ by iterating the mapping

$f_{\theta}:\left\{\begin{aligned} \mathscr{P}(G) & \rightarrow \mathscr{P}(G) \\ M & \mapsto\left\{y \in G: \sum_{x \in M} c \in(x, y) \geqq \theta\right\} .\end{aligned}\right.$

If the set $M$ of neurons is active, this will in turn activate the set $f_{\theta}(M)$ at threshold $\theta$, this in turn will activate $f_{\theta}^{2}(M)$, and so on.

\section{Threshold Control}

The basic idea of threshold control is that the basic neuronal activity evolves rather fast [it takes at most $5 \mathrm{~ms}$ from $M$ to $f_{\theta}(M)$; this is a conservative estimate for the conduction time (axonal and synaptic) of a monosynaptic pathway from one cortical neuron to the next] and that it is controlled by comparatively slow variations of the parameter $\theta$. In other words: in analysing the flow of activity we may keep $\theta$ fixed at first and let the activity evolve through $M, f_{\theta}(M)$, $f_{\theta}^{2}(M), \ldots$ to some final invariant state (or into some cycle). Then we change $\theta$ a bit and look for the new invariant state (that may occasionally be identical to the old one). We can use threshold control to detect cell assemblies: they occur if small variations in $\theta$ do not change the invariant state.

\section{Cycles}

Our graphic model for the dynamics of the brain is too exact in one respect: the activity proceeds from $M$ to $f_{\theta}(M)$ and so on, in discrete time steps. In reality, the activity is "smeared out" in time at every synapse with

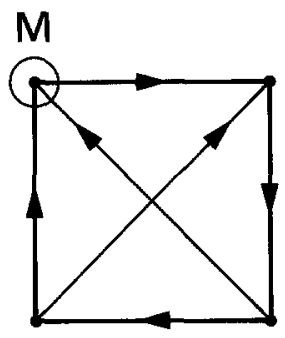

Fig. 5

a time constant of a few milliseconds which is in the same range as the time needed by a monosynaptic pathway. Therefore in the long run cycles at fixed threshold $\theta$ are quite improbable in reality, although they will occur in the model.

For example, in the network of Fig. 4, starting with $M$, the activity will flow around in a cycle forever. In the corresponding real network, the flow of activity will show a cyclic structure at first, but due to the smearing out of activity, the whole net will finally be active.

Thus the real flow of activity in Fig. 4 will be similar to the sequence $M, f_{\theta}(M), f_{\theta}^{2}(M), \ldots$ of Fig. 5 .

For this reason we will not consider cycles as cell assemblies.

\section{Inhibition}

As I said in the first section we may disregard inhibition in the modeling of cell assemblies, since they are formed basically by the excitatory long range cortico-cortical connections.

Before doing so, it seems important to give a rough qualitative picture of the effects of inhibition that we have to keep at the back of our minds in the next section.

\subsection{Specific Inhibition}

This can occur between neighbouring cells in the same cortical area, and it implies that a certain part of one assembly selectively inhibits certain other neurons that are part of another assembly (or several other assemblies). Specific inhibition may for example correspond to the phenomenon that we cannot remember some name, because very often another name that is "too" similar in some respect comes to mind first and prevents the desired name from being remembered.

Specific inhibition may be helpful to define concepts from each other that are very similar in some respect. It may be learned through a synaptic mechanism analogous to Hebb's rule: some local inhibitory connections may be enhanced through common preand postsynaptic activity [for a theoretical analysis of this mechanism - although on a large global scale - see Kohonen (1977, Chap. 3.2)]. 


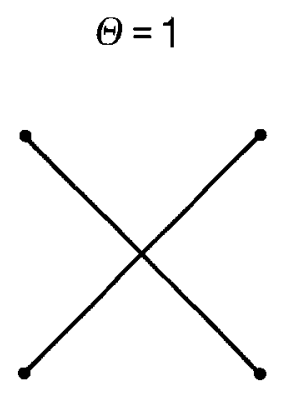

a

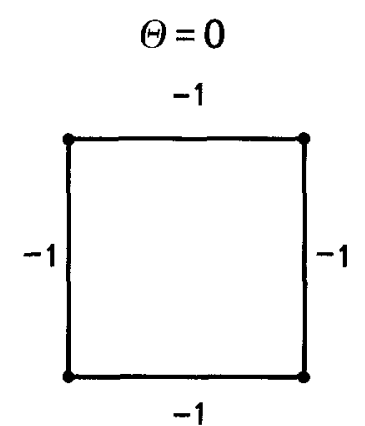

b

Fig. 6. $\mathbf{b}$ is obtained from a by subtracting 1 from the connectivities $c(m, n)$ for $m \neq n$

\subsection{Diffuse Local Inhibition}

At first glance one might believe that a certain level of diffuse inhibition is equivalent to general permanent increase of the threshold $\theta$. This is not true as can be seen in the dynamics of Fig. 6.

Indeed, it turns out that homogeneous diffuse inhibition is equivalent to a "fast" threshold control whereby $\theta$ is increased proportionally to the number of active neurons (large numbers of active neurons distribute more inhibition than small numbers of active neurons).

This fast threshold control has two implications for the number of cell assemblies:

a) It tends to stabilize the number of active neurons, which is good for cell assemblies, since a constant activity (not zero and not total) implies the existence of a cell assembly.

b) It tends to decrease the number of cell assemblies, as can be seen in the network of Fig. 7.

Here we have three obvious assemblies, namely 1 , 2, and 3. But also each combination of two of them, i. e. $(1,2),(1,3),(2,3)$ could be regarded as an assembly since it is invariant and stable against small variations of $\theta$.

However, a certain level of diffuse inhibition would forbid a combination - say $(1,2)$ - of two "elementary" assemblies to stay active, simply because it is twice as large and causes twice as much diffuse inhibition. Therefore we would observe a "competition" between 1 and 2 and only one of them could stay active.

Thus, a certain level of diffuse inhibition will reduce the number of assemblies in the above network from 6 to 3 .

A combination of two assemblies can only be an assembly again if there are some excitatory connections between the two (that help to overcome the increased amount of inhibition).

In the next chapter we shall ignore inhibition, but we incomporate this last requirement into the definition of a cell assembly.
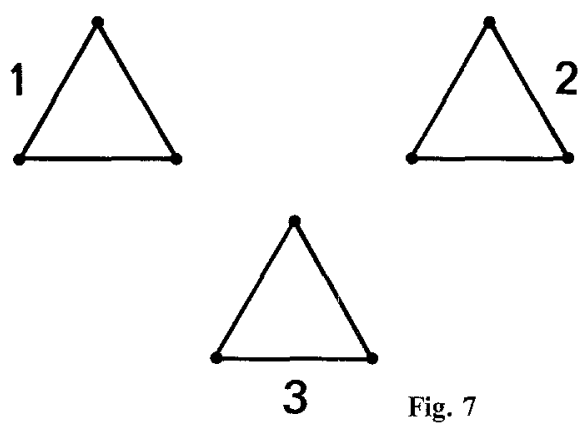

Let me finally discuss the possible psychological interpretation of the effects a) and b).

a) If $M$ denotes a set of neurons (for example an assembly), $|M|$ denotes the number of neurons in the set $M$. The stabilization of $|M|$ may have the side effect that oscillations of $|M|$ around some mean value do occur. This would mean that some quite large part of an assembly is constantly active whereas other parts have just an oscillating activity. I think that these oscillations should not alter the concept represented by the assembly. Since we vary the threshold, we must have a large (for low threshold) and a small (for high threshold) set of neurons to represent the same concept, the small set being contained in the large set. Fluctuation in the size of the assembly may be regarded just as fluctuations in the "loudness" of the corresponding concept, as long as they stay within the boundaries of these two sets.

b) The competition between two concepts is a rather common phenomenon in psychology and psychophysics: think for example of ambiguous figures (Szenthagothai and Arbib, 1974, p. 412) and binocular rivalry (Breese, 1909).

\section{Dynamics at Fixed Threshold}

In this section the number $\theta$ is fixed, and we simply write $f$ instead of $f_{\theta}$. Moreover, we shall assume that $c(x, y) \in \mathbb{N}_{0}$ for every $x, y \in G$.

6.1. Definition. a) $M$ ignites $N$ (at $\theta$ ), if there is a number $n \geqq 1$, such that $N \cong f_{\theta}^{n}(M)$.

b) $M$ exhausts $N$ (at $\theta$ ), if $N \subseteq M^{*}:=\bigcup_{n=0}^{\infty} f_{\theta}^{n}(M)$, i.e. if for every element $x$ of $N$ there is a number $n \geqq 0$, such that $f_{\theta}^{n}(M) \ni x$.

6.2. Definition. A subset $M$ of $G$ is called invariant, if $f(M)=M$

persistent, if $f(M) \supseteqq M$

weak, if there is an $n \in \mathbb{N}: f^{n}(M)=\emptyset$.

6.3. Definition. A sequence $\left(M_{1}, \ldots, M_{l}\right)$ is called a period of length $l$, if $f\left(M_{i}\right)=M_{i+1}$ for $i=1, \ldots, l-1$ and $f\left(M_{l}\right)=M_{1}$. 


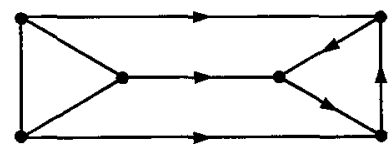

Fig. 8

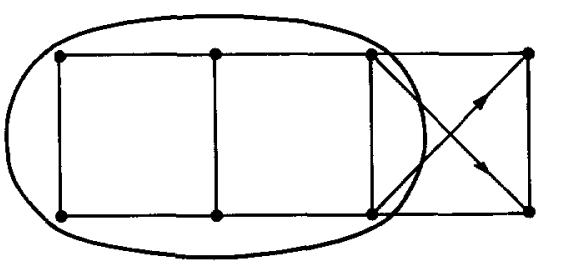

Fig. 9
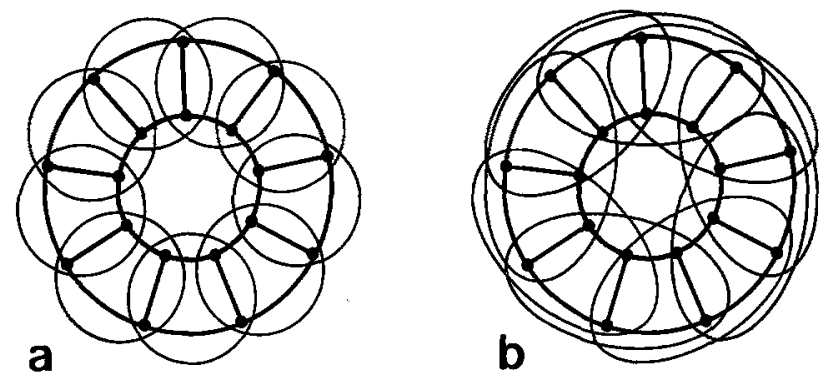

Fig. 10. a shows the 9 minimal persistent sets; b shows the 9 additional tight sets (which are assemblies)

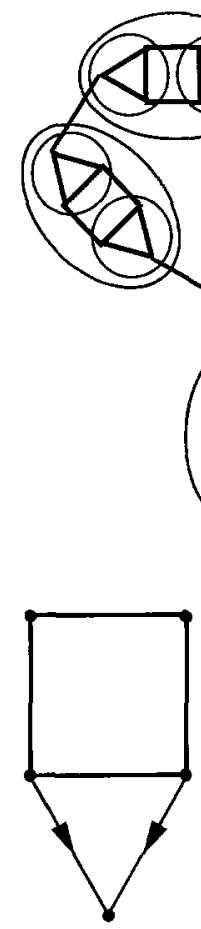

a
Fig. 11. All assemblies are shown

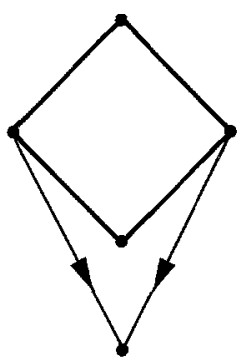

b

Fig. 12
6.4. Proposition. Let $M \subseteq G$. Exactly one of the following statements is true:

a) $M$ is weak,

b) $M$ ignites an invariant set,

c) $M$ ignites a period.
Proof. This follows from the fact that $\mathscr{P}(G)$ is finite.

If $M$ is persistent, we have $M \leqq f(M) \subseteq f^{2}(M) \subseteq \ldots$ and therefore we have case b). The invariant set generated by $M$ is denoted by $\operatorname{cl}(M)$, the closure of $M$.

The next two propositions are obvious.

6.5. Proposition. a) $\operatorname{cl}(\operatorname{cl}(M))=\operatorname{cl}(M)$ for $M$ persistent, b) $A \subseteq B, A, B$ persistent, implies $\operatorname{cl}(A) \subseteq \operatorname{cl}(B)$.

6.6. Proposition. If $A, B$ are persistent, then $A \cup B$ is persistent.

Next, we shall try to define an "assembly" (at $\theta$ ) as a certain subset of $G$ in terms of the dynamics in $G$ at $\theta$.

We shall use the following vague definition: "A cell assembly is a subset $A$ of $G$ such that every sufficiently large subset of $A$ excites all of $A$." Here we have to specify the meaning of "sufficiently large" and "excites".

First of all, we shall require that a cell assembly has to be persistent. But this is not enough: For example, the graph of Fig. 7 is persistent at $\theta=2$, but clearly we would not regard it as an assembly. Thus we could try the following definition of a cell assembly: "every persistent subset ignites the whole set". But this is perhaps a bit too strong. For example, Fig. 8 could well be regarded as an assembly, although the left triangle is persistent and does not ignite the rest. Figure 8 could be regarded as an assembly since the right triangle "needs" the left triangle in order to persist. Therefore we might use the following slightly weaker version : "every persistent subset whose complement is not weak, ignites the whole set". Also this definition is not yet perfect, since an assembly should be closed. This leads us to the final definition of an assembly.

6.7. Definition. a) A persistent set $A$ is called tight, if every persistent subset of $A$ whose complement in $A$ is not weak, ignites the whole of $A$.

b) An assembly is the closure of a tight set. The example of Fig. 9 shows that the closure of a tight set does not need to be tight again:

6.8. Definition. A subset $M$ of a graph $G$ is called minimal persistent, if it is persistent and no proper subset of it is persistent.

6.9. Proposition. Every minimal persistent set is tight.

It is now possible to analyse the structure of weighted graphs (with positive weights) in terms of assemblies, tight sets, and minimal persistent sets. Let me give some examples (Figs. 10 and 11):

Further examples for assemblies are given in the next section. 


\section{Inner Structure of and Relations Between Assemblies}

In this section I shall define some concepts to describe the inner structure of persistent sets, and especially assemblies. We still assume $\theta$ to be fixed and write $f$ instead of $f_{\theta}$. Let $A$ be a persistent set.

We denote by $f_{A}: \mathscr{P}(A) \rightarrow \mathscr{P}(A)$ the mapping $M \vdash f(M) \cap A$.

7.1. Definition. We say that a subset $M$ of $A$ fills $A$, if for every $x \in A$ there is an infinite sequence of numbers $n_{i}$ such that $x \in f_{A}^{n_{i}}(M)$.

For example, in Fig. 12a at $\theta=2$, only the square fills, whereas in Fig. 12b, two opposite points of the square fill as well.

7.2. Definition. a) A subset $M$ of a persistent set $A$ is called a germ of $A$, if it fills $A$ and if no proper subset of $M$ fills $A$.

b) The kernel $K(A)$ of a persistent set $A$ is the union of all germs of $A$.

c) The halo $H(A)$ of a persistent set $A$ is $\operatorname{cl}(A)-K(A)$.

\subsection{Examples (Figs. 13-20)}

7.4. Two assemblies may have an excitatory influence on each other in several different ways. In the following I will give examples for the most important cases I can think of.

a) Intersection. Two assemblies $A$ and $B$ have some neurons in common.

In Fig. 21a and $b$ the two assemblies form a superassembly.

b) Inclusion (Fig. 22)

c) Projection (Fig. 23)

d) Mutual projection (Fig. 24)

In this case $A$ and $B$ together form a superassembly that holds at higher threshold.

\section{Formulation of the Main Problem}

Having defined the notion of an "assembly" we can now formulate precisely the main problem of the theory of cell assemblies: Given a weighted graph $\langle G, c\rangle$ with $c(x, y) \in \mathbb{N}_{0}$ for every $x$ and $y$ in $G$, we may ask for the number $n_{\theta}$ of assemblies at threshold $\theta$ and for the total number of assemblies.

This leads to some very specific graph-theoretical questions that I just want to pose in this section. I have not yet obtained an answer to any of them, but some preliminary results that are hopefully connected to these questions are given in Sect. 10.

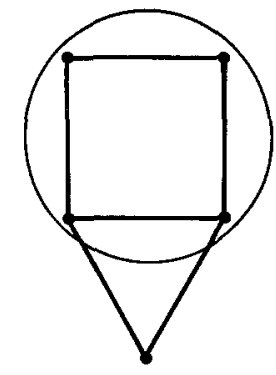

Fig. 13. This figure shows an invariant set; its kernel is circumscribed. The same conventions are used in Figs. $14-20$

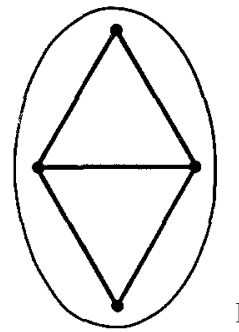

Fig. 14
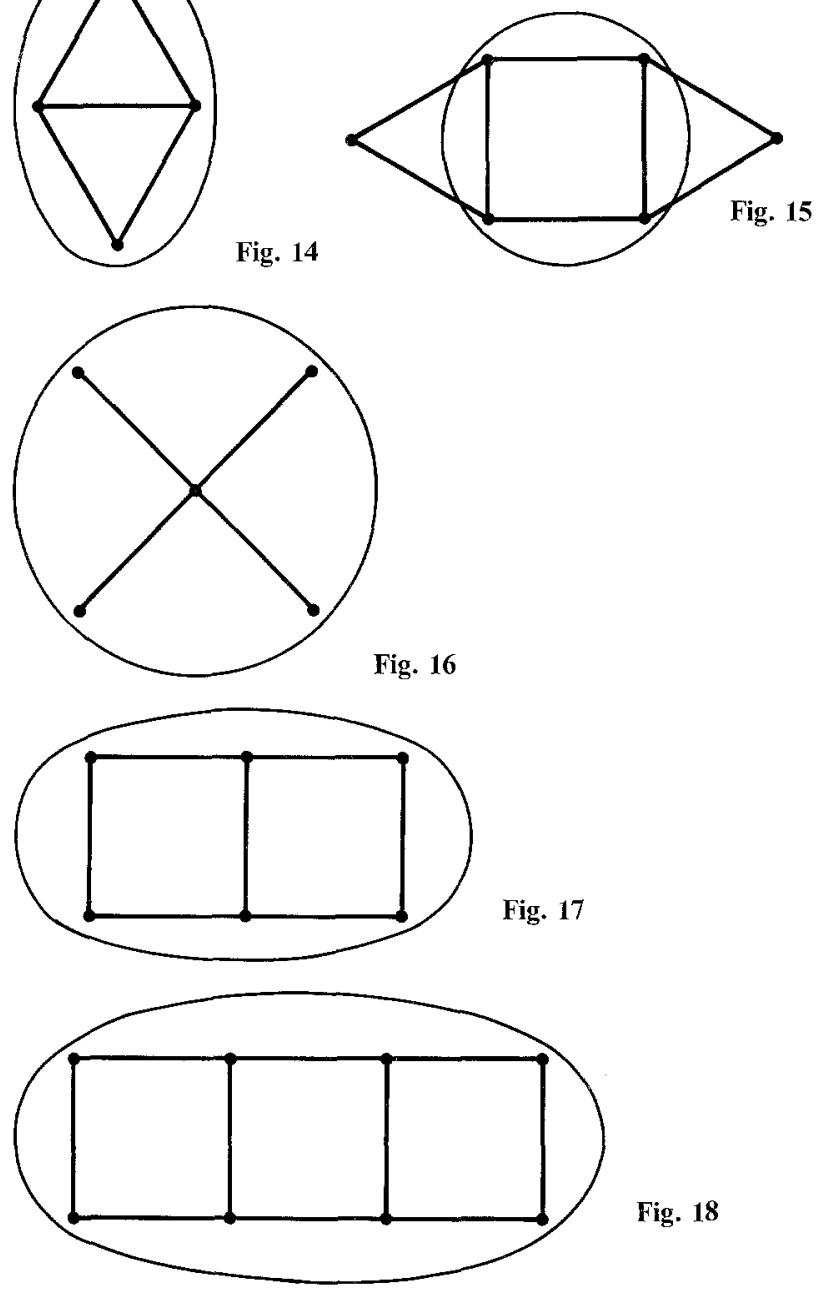

Fig. 18
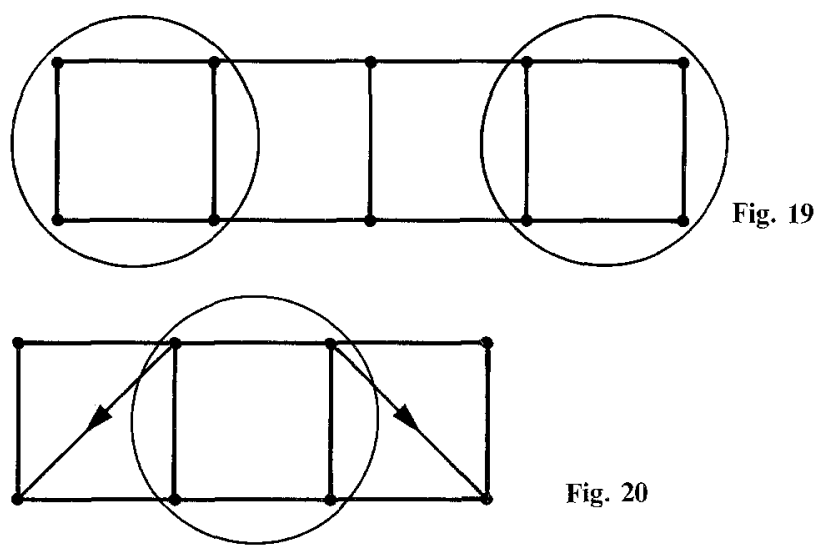

Fig. 20 


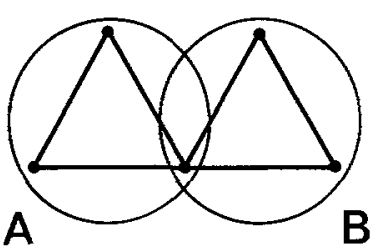

a

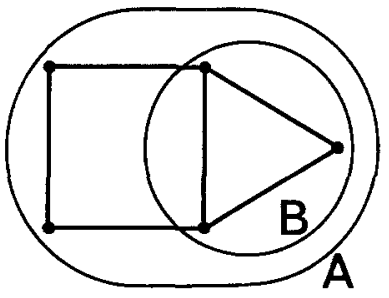

Fig. 22

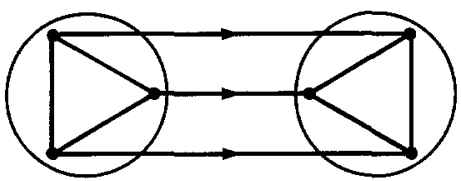

A

B Fig. 23

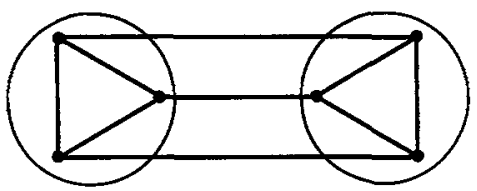

A

B Fig. 24

In the next section I will introduce some further auxiliary concepts (especially notions of connectivity). To pose the questions we need one more definition:

8.1. Definition. A directed graph $\langle G, c\rangle$ is called homogeneous, if for any $x, x^{\prime} \in G \sum_{y} c(x, y)=\sum_{y} c\left(x^{\prime}, y\right)$ and $\sum_{y} c(y, x)=\sum_{y}\left(y, x^{\prime}\right)$ Remark. This implies that for $e:=\frac{1}{|G|} \sum_{x, y} c(x, y)$ and
any $x \in G$ we have

$\sum_{y} c(x, y)=e=\sum_{y} c(y, x)$.

Remember that $|G|$ denotes the number of elements (i.e. points) in $G$.

8.2. Question. Given two numbers $k$ and $a$, determine a weighted graph $\langle G, c\rangle$ with $|G|=k$ and $e=a$ such that $n$ is maximal.

8.3. Question. a) Is it possible to find a homogeneous weighted graph with this property?

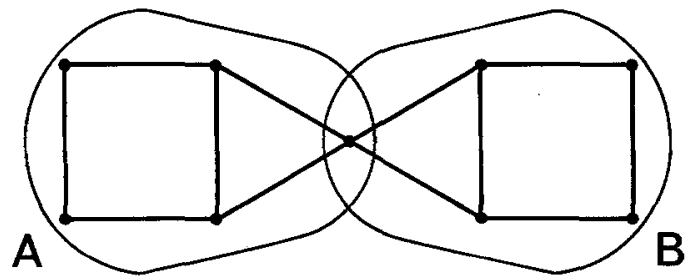

C

Fig. 21

b) Is it possible to find a strongly connected weighted graph with this property? (Notions of connectivity are defined in the next section.)

8.4. Question. How does the function $\theta \mapsto n_{\theta}$ look like, for such a graph?

8.5. Question. Given two numbers $k$ and $a$, consider the set $\mathscr{G}$ of all (homogeneous) (connected) weighted graphs $\langle G, c\rangle$ with $|G|=k$ and $e=a$ and let $\theta>0$. What is the average value for $n_{\theta}$ on the set $\mathscr{G}$ ? What is the average size of an assembly occuring at $\theta$ in a graph in the set $\mathscr{G}$ ?

The last question obviously contains several subquestions, which could even be answered in an asymptotic sense for $k, a \rightarrow \infty$. In this way one could sharpen the vague conjecture that large randomly connected networks contain many assemblies.

\section{Some Characteristic Numbers and Properties of Weighted Graphs}

In this section I shall define and discuss some characteristic numbers associated with weighted graphs, most of which are concerned with the "connectivity" of a graph.

9.1. Definition. $e:=|G|^{-1} \sum_{x, y \in G} c(x, y)$ is called the average connectivity of $G$.

$\theta:=\max \{t: G$ is persistent at $t\}=\min _{y \in G} \sum_{x \in G} c(x, y)$ is called the critical threshold of $G$.

$w c:=\min _{\boldsymbol{A} \subseteq G} \sum_{x \in A} \sum_{y \in A^{c}} c(x, y)$ is called the weak connectivity of $G$.

$c:=\max \left\{t: f_{t}(A) \cap A^{c} \neq \emptyset\right.$ for every $A$ with $|A| \geqq s$ for some $s \leqq t, s<|G|\}$ is called the connectivity of $G$.

Usually $c$ coincides with $c^{\prime}$ which is given by the slightly more intuitive definition:

$c^{\prime}:=\max \left\{t: f_{t}(A) \cap A^{c} \neq \emptyset\right.$ for every $A$ with $\left.|A| \geqq t\right\}$.

Later I shall define some refinements of the definition of $c$. To this end a few somewhat different definitions will be helpful. 
9.2. Definition. $i_{\theta}:=\min \{n:|A| \geqq n$ implies $A \longrightarrow G\}$ is called the ignition number of $G$.

$h_{\theta}:=\min \{n:|A| \geqq n$ implies $A$ holds at $\theta\}$ is called the holding number of $G$.

$i c_{\theta}:=\min \left\{n:|A| \geqq n\right.$ implies $\left.f_{\theta}(A) \supseteqq A^{c}\right\}$ is called the immediate connection number of $G$.

$f_{\theta}:=\min \{n:|A| \geqq n$ implies $A$ fills $G$ at $\theta\}$ is called the filling number of $G$.

$s_{\theta}:=\min \{n:|A| \geqq n$ implies $A$ is not weak $\}$ is called the survival number of $G$.

$d_{\theta}:=\max \{n:|A| \leqq n$ implies $A$ is weak $\}$ is called the dying number of $G$.

$\operatorname{id}_{\theta}:=\max \left\{n:|A| \geqq n\right.$ implies $\left.f_{\theta} A=\emptyset\right\}$ is called the immediate dying number of $G$.

$n_{\theta}:=$ the number of assemblies at $\theta$.

$n:=$ the total number of assemblies.

Usually I shall write $i$ instead of $i_{\theta}$, where $\theta$ is the critical threshold of $G$ (Definition 9.1), and similarly for $h$, etc.

9.3. Proposition. a) $e \geqq \theta$, b) $\theta \geqq w c$ if $c(a, a) \geqq 0$ for every $a \in G$.

Proof. a) $\sum_{y} \sum_{x} c(x, y) \geqq|G| \min _{y} \sum_{x} c(x, y)$.

b) $A=G \backslash\{a\}$ implies

$\sum_{x \in A} \sum_{y \in A^{c}} c(x, y)=\sum_{x \in A} c(x, a) \leqq \sum_{x \in G} c(x, a)$.

Thus $\min _{|\boldsymbol{A}|=G-1} \sum_{x \in A} \sum_{y \in A} c(x, y) \geqq w c$.

9.4. Proposition. a) $h \leqq i c+1$, if $c(a, a) \geqq 0$ for every $a \in G$.

b) $h \geqq i c+1$, if $c(a, a) \leqq 0$ for every $a \in G$.

Proof. a) Let $|A| \geqq i c+1$ and $x \in A$. Then $f(A \backslash x) \ni x$, hence $f(A) \ni x$.

b) Let $|A|=h-1$ and $x \notin A$. Then $f(A \cup\{x\}) \ni x$, hence $f(A) \ni x$.

9.5. Corollary. $i \leqq i c+1$, if $c(a, a) \geqq 0$.

9.6. Proposition. $i \geqq f \geqq s>d \geqq$ id.

Proof. Trivial.

9.7. Definition.

$c_{3}:=\max \left\{t: \quad \operatorname{id}_{t}<|G|\right.$ and $\quad f_{t}(A) \cap A^{c} \neq \emptyset \forall A$ with

$\left.\quad|G|>A>\mathrm{id}_{t}\right\}$.

$c_{2}:=\max \left\{t: \quad \operatorname{id}_{t}<|G|\right.$ and $\quad A_{t}^{*} \supseteqq A^{c} \quad \forall A$ with

$c_{1}=\max \left\{t: \quad \mathrm{id}_{t}<|G|\right.$ and $\left.A \mid>A>\mathrm{id}_{t}\right\}$.

$c_{1}:=\max \left\{t: \quad \operatorname{id}_{t}<|G|\right.$ and $A$ fills $A^{c}$ at $t \forall A$ with

$c_{0}:=\max \left\{t: \quad \operatorname{id}_{t}<|G|\right.$ and $\quad f(A) \supseteq A^{c} \quad \forall A$ with

$\left.|G|>A>\mathrm{id}_{t}\right\}$.
9.8. Proposition. a) $c_{3} \geqq c_{2} \geqq c_{1}$

b) $\theta \geqq c$, if $c(a, a) \geqq 0$

Proof. a) Trivial.

b) We have to show that $G$ is persistent at $c$ : for $x \in G$, $f_{c}(G \backslash\{x\}) \ni x$.

9.9. Proposition. Let $\mathrm{id}_{t}<|G|$.

a) If $c(x, y) \in \mathbb{Z}$, then $\mathrm{id}_{t} \leqq t-1$.

b) If $c(x, y) \leqq 1$, then $\mathrm{id}_{t} \geqq t-1$.

Proof. $\mathrm{id}_{t}<|G|$ means that there is an $x \in G$ and $M \subseteq G$ such that $\sum_{y \in M} c(y, x) \geqq t$.

a) Let $A:=\{y: c(y, x)>0\}$ then $\sum_{y \in A} c(y, x) \geqq t$. If $|A| \leqq t$ we are done, if $|A| \geqq t$ any subset $B$ of $A$ with $|B|=t$ will satisfy $\sum_{y \in B} c(y, x) \geqq t$.

b) If $|M| \leqq t-1$ and $x \in G$ then $\sum_{y \in M} c(y, x) \leqq t-1$.

9.10. Corollary. a) If $c(x, y) \in \mathbb{Z}$ for every $x, y \in G$, then $c \geqq c_{3}$.

b) If $c(x, y) \leqq 1$ for every $x, y \in G$, then $c \leqq c_{3}$.

Proof. Follows from 9.9 .

9.11. Proposition. Let $c(x, y) \in\{0,1\}$ for every $x, y \in G$.

a) $c_{3}=c \leqq w c$.

b) Let $\vartheta \leqq c$, then $|A| \geqq \vartheta$ implies $f_{\vartheta}(A) \cap A^{c} \neq \emptyset$.

Proof. a) By 9.10, we have $c_{3}=c$.

Let $A \subseteq G$, we have to show $\sum_{x \in A} \sum_{y \in A^{c}} c(x, y) \geqq c$. If $|A| \geqq c$, then $f_{c}(A) \cap A^{c} \neq \emptyset$, and therefore

$\max _{y \in A^{c}} \sum_{x \in A} c(x, y) \geqq c$.

If $|A|=: m<c$, let $\sum_{x \in A} \sum_{y \in A^{c}} c(x, y)=: k$. Assume that $k<c$.

Take $B \supseteqq A$ such that $|B|=c$ and $B$ contains as many elements of $f_{1}(A) \backslash A$ as possible.

Take $x \in B^{c}$, then $\sum_{b \in B \backslash A} c(b, x) \leqq|B \backslash A|=c-m$,

$\sum_{b \in A} c(b, x) \leqq\left\{\begin{array}{llr}k-(c-m) & \text { if } & \left|f_{1}(A) \backslash A\right| \geqq c-m \\ 0 & \text { if } & \left|f_{1}(A) \backslash A\right| \leqq c-m,\end{array}\right.$

and therefore $\sum_{b \in B} c(b, x) \leqq\left\{\begin{array}{c}k \\ c-m\end{array}\right\}<c$.

This shows $f_{c}(B) \cap B^{c}=\emptyset$.

b) Here the argument is similar to a), but simpler; it is left to the reader. 


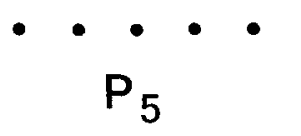

a

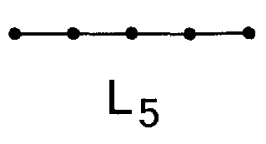

b

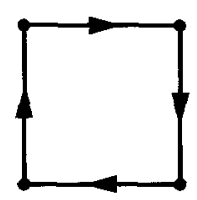

$\mathrm{DC}_{4}$

e

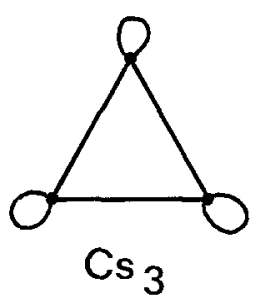

b

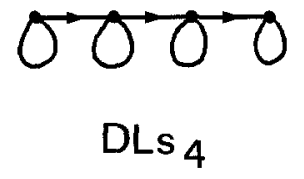

a

Fig. 26. a shows $D L s_{4} ;$ b shows $C s_{3}$

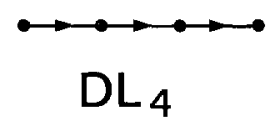

C

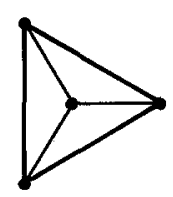

K 4 Fig. 25. a shows $P_{5}$; b shows

$L_{5} ; \mathbf{c}$ shows $D L_{4} ; \mathbf{d}$ shows

$C_{6} ;$ e shows $D C_{4} ; \mathbf{f}$ shows

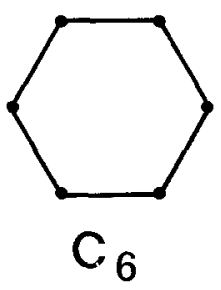

d

9.12. Proposition. Let $c(x, y) \in \mathbb{Z}$ for every $x, y \in G$ and $w c \geqq 1$, then $c, c_{3}, c_{2}, c_{1} \geqq 1$. (In this case the graph $G$ is called connected.)

Proof. In view of 9.10 and 9.8 , we have to show $c_{1} \geqq 1$. It is sufficient to show that for $x, y \in G$ there is an $n \in \mathbb{N}$ such that $f_{1}^{n}(x) \ni y$. [Indeed, this implies $f_{1}^{m}(y) \ni x$ for some $m \in \mathbb{N}$ and therefore $f_{1}^{2 n+m}(x) \supseteqq f_{1}^{n+m}(y) \supseteqq f_{1}^{n}(x) \ni y$, etc.]

Thus we have to show that $\{x\}_{1}^{*}=G$ for any $x \in G$. Assume $A:=\{x\}_{1}^{*} \neq G$, then

$f_{1}(A) \leqq A$ implies $\sum_{x \in A} \sum_{y \in A^{c}} c(x, y) \leqq 0$,

in contradiction to $w c \geqq 1$.

9.13. Proposition. Let $c(x, y) \in \mathbb{Z}$ for every $x, y \in G$.

If $c_{0} \leqq \frac{|G|}{2}$, then $c_{1} \geqq c_{0}$.

Proof. Let $|A|=\mathrm{id}_{c_{0}}+1 \leqq c_{0}$

Then $\left|A^{c}\right| \geqq|G|-c_{0} \geqq \frac{|G|}{2} \geqq c_{0} \geqq \mathrm{id}_{c_{0}}+1$

Therefore $f_{c_{0}}\left(A^{c}\right) \supseteqq A$ and $f_{c_{0}}(A) \supseteqq A^{c}$.

Thus $A$ fills $G$ at $c_{0}$.

This is a fortiori true for any $B$ with $B \supseteqq A$. This means that $|B|>\mathrm{id}_{c_{0}}$ implies that $B$ fills $G$ at $c_{0}$.

\section{Construction of Graphs and Examples}

Let me start this section by introducing some general graphs and some general ways of constructing new graphs from given ones.

10.1. Definition. 1) $P_{n}$ denotes the graph $G$ containing $n$ separate points (Fig. 25a).

2) $L_{n}$ denotes a single line containing $n$ points (Fig. 25b).

2) $D L_{n}$ denotes a directed line containing $n$ points (Fig. 25c).

3) $C_{n}$ denotes a circle with $n$ points (Fig. $25 \mathrm{~d}$ ).

3') $D C_{n}$ denotes a directed circle with $n$ points (Fig. 25e).

4) $K_{n}$ denotes the complete graph with $n$ points (Fig. 25f).

10.2. Definition. The suffix $s$ adds selfconnections to the graphs $P_{n}, L_{n}, K_{n}, C_{n}, D L_{n}$, and $D C_{n}$ (compare Fig. 26).

10.3. Given any two directed graphs $H$ and $G$ we may construct the following graphs (compare Fig. 27):

1) $\tilde{G}$ has connections exactly where $G$ has not, but $\tilde{G}$ does not have self connections.

2) $G+H$ means that $G$ and $H$ are placed side by side without any connections.

3) $G+{ }_{c} H$, called the connected sum (or join): $G$ and $H$ are placed side by side and every point of $G$ is connected (both ways) with every point of $H$.

4) $G+{ }_{d} H$, called the directed sum : $G$ and $H$ are placed side by side and there is a connection from every point of $G$ to every point of $H$. 


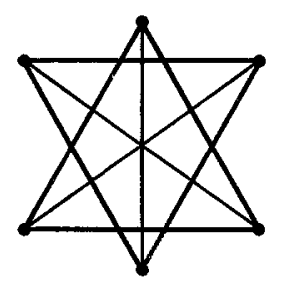

a

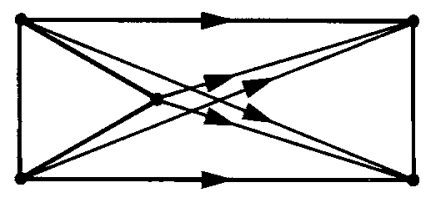

d

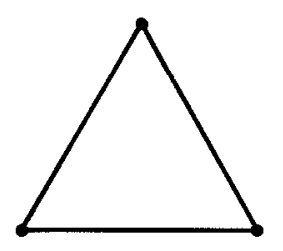

Fig. 28. $e=2, \theta=2, w c=2, c=1$

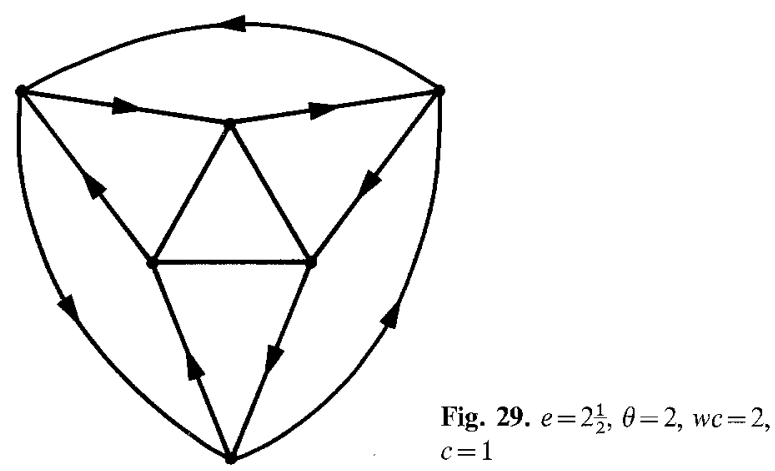

5) $G \times H$ : Every point of $G$ is replaced by one version of $H$ and every arrow of $G$ is replaced by $|H|$ arrows connecting the corresponding points of the two versions of $H$ that have replaced the two endpoints of the arrow.

10.4. Some exercises with these notions:

a) $K_{2}=L_{2}$.

b) $K_{3}=C_{3}$.

c) $\tilde{C}_{4}=L_{2}+L_{2}$.

d) $C_{4}=K_{2} \times K_{2}$.

e) $\tilde{C}_{5}=C_{5}$.

f) $\tilde{C}_{6}=K_{3} \times K_{2}$.

g) $P s_{1} \times C_{n}=C s_{n}$.

h) $P s_{2} \times C_{n}=C s_{n}+C s_{n}$.

i) $P_{3} \times G=G+G+G$ for any graph $G$.

j) $P_{i}+P_{j}=P_{i+j}$.

k) $P_{i} \times P_{j}=P_{i, j}$.

1) $K_{i}+{ }_{c} K_{j}=K_{i+j}$.

m) $G \times H=H \times G$ for any two graphs $G$ and $H$.

e
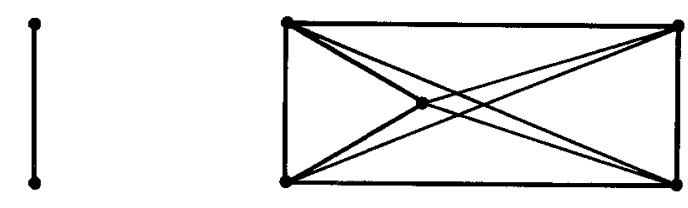

C

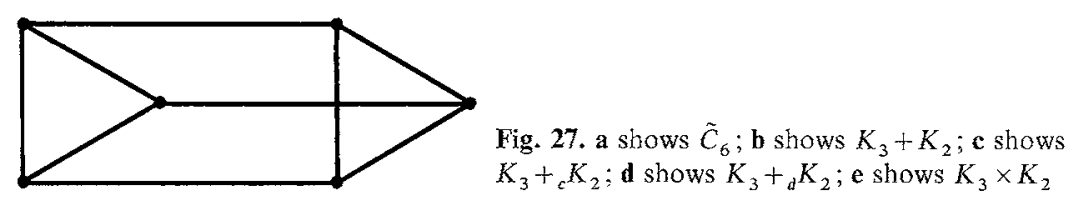

n) $G+{ }_{c} H=H+{ }_{c} G$ for any two graphs $G$ and $H$.

o) $\overline{G+}{ }_{d} H=\tilde{H}+{ }_{d} \tilde{G}$ for any two graphs $G$ and $H$.

p) $\overline{G+{ }_{c} H}=\tilde{G}+\tilde{H}$ for any two graphs $G$ and $H$.

10.5. Let me finally give the connectivities as defined in the last section for some specific graphs (Figs. 28-33).

$$
\begin{array}{rlrl}
L_{n}: e & =2 \frac{n-1}{n} & \theta & =1, w c=1 \\
D L_{n}: e & =\frac{n-1}{n} & \theta & =0, w c=0 \\
C_{n}: e=2 & \theta & =2, w c=2, c=1 \\
D C_{n}: e=1 & \theta & =1, w c=1 \\
K_{n}: e & =n-1 & \theta & =n-1, w c=n-1, c=c_{3}=n-1, \\
c_{2}=n-1, c_{1}=\left[\frac{n}{2}\right] & \\
\tilde{C}_{n}: e & =n-3 & \theta & =n-3, w c=n-3, c=c_{3} \approx \frac{n}{3}, \\
c_{2} & =?, & c_{1} & =?
\end{array}
$$

$C_{n} \times L_{2}: e=3 \quad \theta=3, w c=3, c=1$

$L_{n} \times L_{2}: e=\frac{3 n-2}{n}, \theta=2, w c=2, c=1$.

\section{On the Construction of Graphs with Many Assemblies and/or High Connectivity}

Let us take $|G|=6$ and let us try to construct a graph with as many assemblies as possible. Of course, we hope that we should be able to get more than the 6 assemblies of the rather trivial construction $P_{s_{6}}$ (Fig. 34):

For example we could try $C s_{6}$ (Fig. 35):

This graph is homogeneous and connected and it has 6 assemblies consisting of the 6 pairs of neighbouring points and 6 further assemblies consisting of the 6 triplets of neighbouring points. A set of 4 neighbouring points is no longer an assembly, since it is "combined of" two pairs of neighbouring points. 


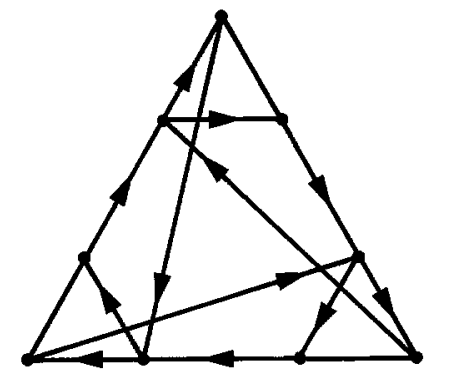

Fig. 30. $e=2, \theta=2, w c=2$, $c=1$

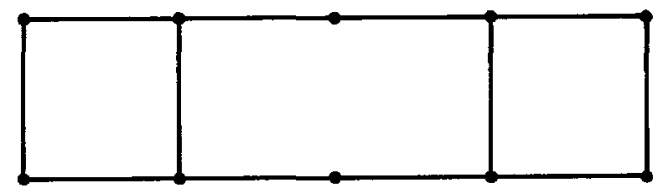

Fig. 31. $e=2.4, \theta=2, w c=2, c=1$

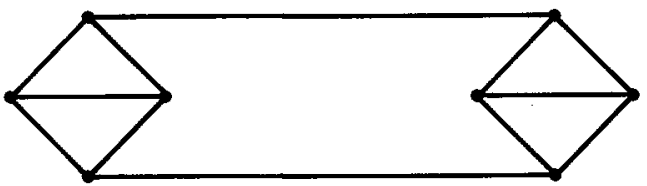

Fig. 32. $e=3, \theta=3, w c=2, c=1$

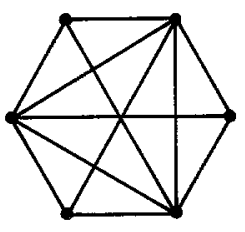

Fig. 33. $e=4, \theta=3, w c=3, c=c_{3}=3$, $c_{2}=2, c_{1}=2$
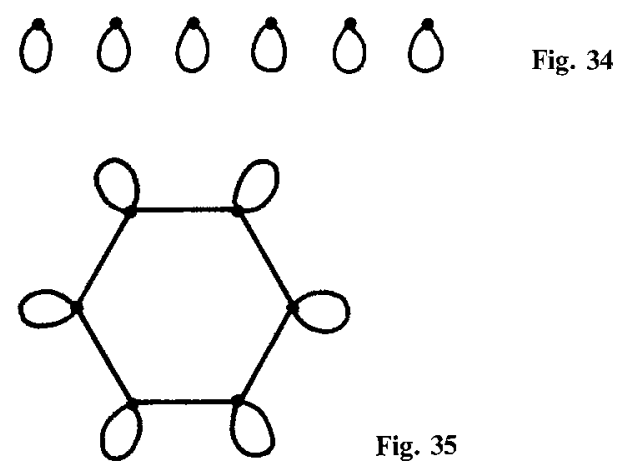

Fig. 35

The following weighted graph contains both the assemblies of $P s_{6}$ and those of $C s_{6}$ : see Fig. 36 .

But using this strong self-excitation we can get a rather trivial solution to the problem, namely the graph $G_{1}$ obtained from $K_{6}$ by adding self excitation of the strength $c(i, i)=2(i=1, \ldots, 6)$ : see Fig. 37 .

In this graph every set of points forms an assembly, since any set of $k$ points is minimal persistent and invariant at $\theta=k+1$. Thus, $n=2^{6}-1=63$.

In the following it will be more interesting to forbid selfconnections or to work only with directed graphs (i.e. to forbid numbers on the arrows). I will now

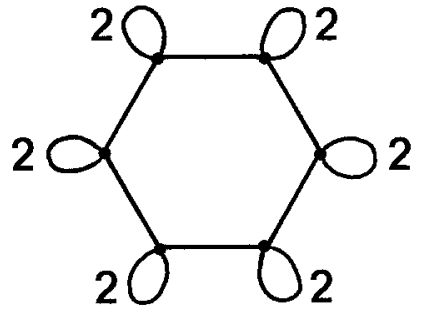

Fig. 36

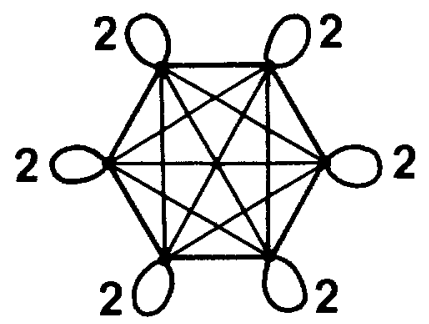

Fig. 37

restrict myself to directed graphs without selfconnections.

The following general strategy for constructing graphs with many assemblies may have emerged from the previous discussion: Use the above results with self-connectivity. Replace every point in a "good" graph $G$ with self-connectivity of strength 1 (like $C s_{6}$ ) by one exemplar of $K_{2}$, in other words: try $C_{6} \times K_{2}$.

Replace every point in a "good" graph $G$ with selfconnectivity of strength 2 (like $G_{1}$ above) by one exemplar of $K_{3}$, in other words: try $K_{6} \times K_{3}$.

For $|G|=6$, this strategy yields $P_{3} \times K_{2}$ with $n=n_{1}=3$ and $K_{2} \times K_{3}$ with $n=3$.

Furthermore I would like to mention the graph of Fig. 38 with $n=n_{2}=1$ :

Up to now I could not find a graph with $|G|=6$ and $n>3$. At first sight this may look rather pessimistic but for larger $|G|$ the situation becomes much better:

Let me just show some graphs $G$ with many assemblies for $|G|=36$. From the first strategy, we may try $K_{12} \times K_{3}$ with $n \geqq 2^{12}$ or for example $G_{2} \times K_{2}$ where $G_{2}$ is given in Fig. 39. Here we get $n \geqq 2+8 \cdot 18=146$ :

Let me give two more graphs: $L_{6} \times L_{6}$ with $n=81$ (all at $\theta=2$ ) and $C_{6} \times C_{6}$ with $n=99$ (where we get the 18 additional assemblies at $\theta=3$ ).

The champion among all these graphs is $K_{12} \times K_{3}$, a homogeneous graph with high connectivity $(w c=13$, $c=2$ ). This graph, however, needs quite a number of connections: the graph $K_{n} \times K_{m}$ has $e=n+m-2$.

Therefore, if we restrict the average number $e$ of connections per point, graphs of the type $K_{n} \times K_{m}$ are certainly not the best ones : for example $K_{6} \times K_{6}$ has $e=10$ and probably $n=125$ which is not as good as the example $G_{2} \times K_{2}$ with $n \geqq 146$ and $e=5$. 
But still, the graphs $K_{n} \times K_{m}$ do illustrate that it is possible to contruct large graphs with a very large number of assemblies:

The graph $K_{n} \times K_{m}$ has $n \cdot m$ points, $(n \cdot m) \cdot(n+m-2)$ connections, i.e. $e=n+m-2$, and at least $2^{n}+2^{m}-4$ assemblies.

Let me close these considerations at this still very unsatisfactory state and let me add that I was quite surprised to get $n$ as high as $2^{12}$ for $|G|=36$ and that I am very curious to know the maximum value for $n$ in this case.

\section{Applications in other Areas}

In this section I only want to hint at possible applications of well-constructed graphs with many assemblies in two other areas of research:

One is the problem of providing connectivity schemes for associative matrix memories.

The other is the problem of designing decentralized communication networks.

12.1. In Palm (1980) I have described associative matrix memories and especially the case of autoassociation.

In the case of auto-association a 0,1 -sequence $s$ of length $n, s=\left(s_{1}, \ldots, s_{n}\right)$ (called a "pattern") is "stored" in a 0,1 -matrix by forming $s \otimes s=\left(s_{i} \cdot s_{j}\right)_{i, j}$.

A set $\mathscr{S}=\left\{s^{1}, \ldots, s^{z}\right\}$ of such patterns is "stored" in the matrix

$A=A_{\mathscr{S}}=\max _{k=1}^{z} s^{k} \otimes s^{k}$.

If we interprete the matrix $A$ as the connectivity matrix of a graph $G_{A}$ with $n$ points, we see that each pattern $s^{k}$ is stored by connecting all the points of $G_{A}$ that correspond to those places where the 0,1 -sequence $s^{k}=\left(s_{1}^{k}, \ldots, s_{n}^{k}\right)$ contains a " 1 ", to a complete subgraph of $G_{A}$.

If these subgraphs do not overlap too much, they will form assemblies and therefore it will be possible to reconstruct the original patterns $s_{1}, \ldots, s_{z}$ from $G_{A}$ by detecting assemblies in $G_{A}$.

The matrix $A$ is called the auto-association matrix for the set $\mathscr{S}$ of patterns.

12.2. Now the problem is the following: Does this scheme still work if not every two points in $G_{A}$ can be connected?

The answer to this question obviously depends on the restrictions that are given to the possibility of connections.

These restrictions are given in a connectivity scheme C. $C$ is an $n \times n 0,1$-matrix, where $c_{i j}=1$ means that $i$ can be connected to $j$ and $c_{i j}=0$ means that it cannot.

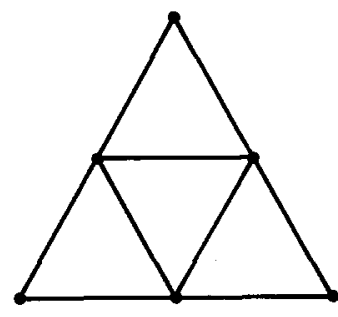

Fig. 38

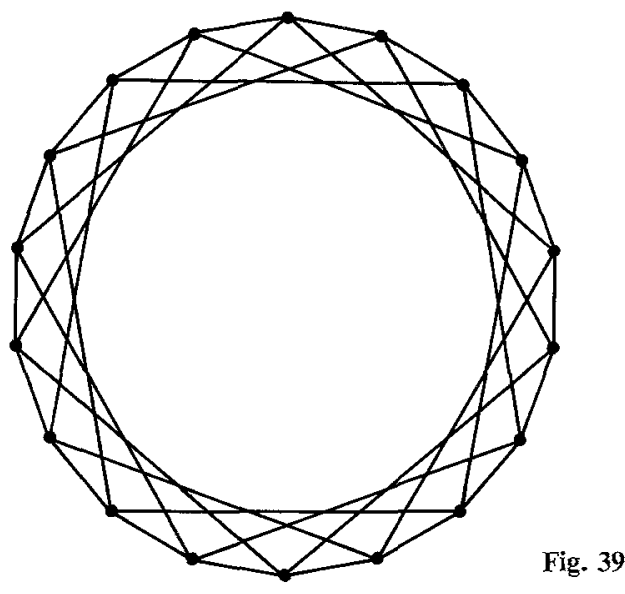

Of course the restriction can as well be described by the directed graph $G_{c}$ corresponding to $C$. Given a set $\mathscr{S}$ of patterns, we now form the matrix $C_{\mathscr{f}}:=A_{\mathscr{S}} \cdot C$, where $(X \cdot Y)_{i j}:=x_{i j} \cdot y_{i j}$ and the corresponding subgraph $G$ of $G_{c}$. Then we try to reconstruct the patterns in $\mathscr{I}$ by detecting the assemblies in $G$. Obviously, if $G_{c}$ is the complete graph $K s_{n}$, we have no restriction to the connectivity and we are in Sect. 12.1.

But can we design a connectivity scheme $C$ with not too many connections such that the modified procedure still works?

12.3. To this end, we may assume that each pattern in $\mathscr{P}$ contains $k$ ones. Then we should try to design a matrix $C$, or a graph $G_{c}$ with small $e$ and $h_{t} \leqq k$ for a high value of $t$. This means that $i c_{t} \leqq k-1$, since $h_{t}=i c_{t}+1$ (Proposition 9.4). In fact, an optimal graph for this kind of problem can be found, but the solution turns out to require a discouragingly high number $e$ of connections.

Let us try to construct a directed graph $G$ with $|G|=n$ and $i c_{t} \leqq k-1$. This means that for every subset $S$ of $G$ containing $k$ points and every point $x \notin S$ there are at least $t$ connections from $S$ to $x$ (this implies $k \geqq t$ ).

Let us look at the complementary graph $\tilde{G}$ : For every subset $S$ of $\tilde{G}$ with $k$ points and every point $x \notin S$ there are at most $k-1-t$ connections from $S$ to $x$. This means exactly that every point $x \in \tilde{G}$ receives at most $k-1-t$ connections. Since we wanted to construct $G$ with as few connections as possible, we have to construct $\tilde{G}$ with as many connections as possible. Thus in the optimal graph $\tilde{G}$, every $x \in \tilde{G}$ receives exactly $k-1-t$ connections. Therefore any homo- 
geneous graph $\tilde{G}$ with $e=k-1-t$, and any homogeneous graph $G$ with

$e=(n-1)-(k-1-t)=n+t-k$

is optimal.

12.4. The second problem that I announced for this section is the following:

Given $n$ "participants" find a communication network for them with a minimal number of links which connects any two participants via not too many links.

12.5. Definition. For $M, N \leqq G$ we define

$t_{9}(M, N):=\min \left\{i: f_{9}^{i}(M) \cap N \neq \emptyset\right\}$.

Then we define

$t_{\vartheta}(k):=\max \left\{t_{9}(M, N):|M|=k,|N|=1\right\}$.

Then $l:=t_{1}(1)$ denotes the number of links through which any two participants can be connected.

The problem can now be exactly formulated: Given two numbers $n$ and $k$. Find a (homogeneous) graph $G$ with $|G|=n$ and $l=k$, such that $e$ is minimal. Moreover, find a graph $G$ with the additional property that $k$ does not increase (too much) if any point is removed from $G$.

The additional property (as well as the homogeneity requirement) is meant to represent the requirement that the network should be decentralized.

I believe that this problem is somehow related to the other problem of this section and also to the problem of cell assemblies, and the following theorem hints in this direction.

12.6. Theorem. Let $G$ be a graph with $|G|=n$ and suppose that $t_{\vartheta}(k)=1$ (i.e. $i c_{\vartheta} \leqq k$ ) for two numbers $\vartheta$, $k \in \mathbb{N}$ with

$\vartheta \leqq k \leqq \frac{n+\vartheta^{2}+1}{\vartheta+1}$.

Then $t_{\vartheta}(\vartheta) \leqq 2$.

12.7. Remark. This theorem can also be formulated as follows :

$i c_{\vartheta} \leqq \frac{|G|+\vartheta^{2}+1}{\vartheta+1}$ implies $t_{\vartheta}(\vartheta) \leqq 2$.

And for $\vartheta=1: i c_{1} \leqq \frac{|G|+2}{2}$ implies $l \leqq 2$.

12.8. Remark. Figure 30 shows a graph with $l=3$ and a low number of connections $(e=2)$. However, removing one point from this graph, may increase $l$ to 5 .
Acknowledgements. I wish to thank V. Braitenberg for many helpful discussions, A. Schütz for careful reading of the manuscript, and L. Ribi for the preparation of the figures.

\section{References}

Amari, S.: A method of statistical Neurodynamics. Kybernetik 14, 201-215 (1974)

Amari, S., Yoshida, K., Kanatani, K.: A mathematical foundation for statistical neurodynamics. SIAM J. Appl. Math. 33, 95-126 (1977)

Braitenberg, V.: On the representation of objects and their relations in the brain. In: Physics and mathematics of the nervous system. Conrad, M., Güttinger, W., Dal Cin, M. (eds.) p. 290. Berlin, Heidelberg, New York: Springer 1974

Braitenberg, V.: Cortical architectonics: general and areal. In: Architectonics of the cerebral cortex. Brazier, M.A.B., Petsche, H. (eds.) p. 443. New York: Raven Press 1978

Braitenberg, V.: Cell assemblies in the cerebral cortex. In : Theoretical approaches to complex systems. Heim, R., Palm, G. (eds.), p. 171. Berlin, Heidelberg, New York: Springer 1978

Breese, B.B. : Binocular rivalry. Psychol. Rev. 16, 410-415 (1909)

Collins, A.M., Loftus, E.F.: A spreading-activation theory of semantic processing. Psychol. Rev. 82, 407-428 (1975)

Cowan, J.D. : Are there modifiable synapses in the visual cortex? In: Neural mechanisms of learning and memory. Rosenzweig, M.R., Bennett, E.L. (eds.), p. 133. Cambridge MA : MIT Press 1976

Harari, F.: Graph theory. Reading MA: Addison-Wesley 1969

Harth, E.M., Csermely, T.J., Beek, B., Lindsay, R.D.: Brain functions and neural dynamics. J. Theoret. Biol. 26, 93-120 (1970)

Hebb, D.O.: The organization of behavior. New York: Wiley 1949

Hebb, D.O.: Textbook of psychology. Philadelphia, London, Toronto: Saunders 1958

Kohonen, T.: Associative memory. Berlin, Heidelberg, New York: Springer 1977

Legendy, C.R.: On the scheme by which the human brain stores information. Math. Biosci. 1, 555-597 (1967)

Legendy, C.R.: How large are Hebb's cell assemblies? In: Cybernetic problems in bionics. Oestreicher, H.L., Moore, D.R. (eds.), p. 721. New York: Gordon and Breach 1968

Palm, G. : On associative memory. Biol. Cybern. 36, 19-32 (1980)

Palm, G., Braitenberg, V.: Tentative contributions of neuroanatomy to nerve net theories. In: Progress in cybernetics and systems research. Trappl, R., Klir, G.J., Ricciardi, L. (eds.), Vol. 3, p. 369. London: Advance Publ. 1979

Quilian, M.R.: Word concepts: a theory and simulation of some basic semantic capabilities. Behav. Science 12, 410-430 (1967)

Quilian, M.R.: Semantic memory, In: Semantic information processing. Minsky, M. (ed.). Cambridge MA: MIT Press 1968

Szenthagothai, J., Arbib, M.A. : Conceptual models of neural organization. NRP Bull. 12, 3 (1974)

Received: August 2, 1980

Dr. G. Palm

Max-Planck-Institut

für biologische Kybernetik

Spemannstrasse 38

D-7400 Tübingen

Federal Republic of Germany 